\title{
Triple pomeron vertex in the limit $N_{c} \rightarrow \infty$.
}

\author{
M.Braun $^{a, b}$ and G.P.Vacca ${ }^{a}$ \\ ${ }^{a}$ Department of Physics, University of Bologna and INFN, Italy, and \\ ${ }^{b}$ Department of High Energy Physics, University of S.Petersburg
}

October 1997

\begin{abstract}
In the hard pomeron theory with the number of colours $N_{c} \rightarrow \infty$ the diffractive amplitude obtained in [3] is compared with the results found for $N_{c}=3$ in [1] and in the dipole approach in [5]. It is shown that the double pomeron exchange contribution can be substituted by an equivalent triple pomeron interaction term. After such a substitution the triple pomeron vertices in $[1,3,5]$ essentially coincide. It is demonstrated that, in any form, the triple pomeron vertex is conformal invariant. It is also shown that higher order densities in the dipole approach do not involve 1 to $\mathrm{k}$ pomeron verteces with $k>2$ but are rather given by a set of pomeron fan diagrams with only a triple pomeron coupling.
\end{abstract}




\section{Introduction}

In the hard pomeron theory the first step towards unitarization goes through the construction of the amplitude generated by the exchange of four reggeized gluons. In the old Regge-Gribov theory this amplitude was a sum of the double pomeron exchange (DPE) and triple pomeron interaction (TPI) contributions, both having essentially the same behaviour at high energies. For a realistic case of the number of colours $N_{c}=3$ the four-gluon system was studied by J.Bartels and M.Wuesthoff [1]. They obtained a complicated system of coupled equations for different colour channels amplitudes. The inhomogeneous terms for this system included a structure which was interpreted as a triple-pomeron vertex. Its explicit expression, found in [1] is rather complicated (it consists of 19 terms). Later it was demonstrated that the found vertex was conformal invariant [2]. In a recent publication [3] we repeated the derivation of [1] in the limit $N_{c} \rightarrow \infty$ guided by the idea that in this limit the leading contribution reduces to a single BFKL pomeron [4]. We found that in the limit $N_{c} \rightarrow \infty$ the complicated system of J.Bartels and M.Wuesthoff decouples and can be explicitly solved both in the leading and subleading approximations in $1 / N_{c}$. The leading contribution indeed reduces to a single BFKL pomeron exchange, as expected. The subleading diffractive amplitude was found to be a sum of two terms, the DPE and TPI, in full correspondence with the Regge-Gribov picture. However the found triple pomeron vertex (also quite complicated) resulted different from the one obtained by J.Bartels and M.Wuesthoff. Also its conformal properties remained unclear. On the other hand, at the same time R.Peschanski calculated the double dipole density in the A.Mueller dipole approach [5], valid in the $N_{c} \rightarrow \infty$ limit. From his result he extracted the triple pomeron vertex, which is rather simple and superficially different from the ones discussed above, obtained via the $s$-channel unitarity approach. No contribution which could be interpreted as the DPE seems to appear in the dipole approach.

Given the variety of the expressions for the 4-gluon diffractive amplitude and the triple pomeron vertex, we dedicate this note to compare these different results in the $N_{c} \rightarrow \infty$ limit. Our main conclusion is that, in fact, they essentially coincide, since, as will be explicitly demonstrated, the DPE contribution can be substituted by a completely equivalent TPI term (but not vice versa). Once this is done, our vertex found in [3] coincides with the one in [1], provided one takes the limit $N_{c} \rightarrow \infty$. Coupled to pomerons, this vertex effectively reduces to the one found by R.Peschanski in [5]. However this does not mean that the double dipole density in the dipole approach coincides with the diffractive amplitude in the $s$-channel unitarity approach: there are certain terms in the latter which are missing in the double dipole density.

As a byproduct of our study we prove that the triple pomeron vertex found in [3] is also conformal invariant. We also comment on the higher- order dipole densities in A.Mueller's approach, in relation to the form of $1 \rightarrow k$ pomeron vertex proposed by R.Peschanski in [5].

The contents of this note is distributed as follows. In Sec. 2, of an introductory character, we present a generalization of the 4-gluon amplitude in the $N_{c} \rightarrow \infty$ limit to a non-forward direction, necessary to study its conformal properties. Sec. 3 is devoted to these properties.

In Sec. 4 we demonstrate the equivalence of the DPE and certain TPI terms. In Sec.5 we compare the 4-gluon diffractive amplitudes found in different approaches. In Sec. 6 we briefly discuss the higher order dipole densities. Finally Sec. 7 contains some conclusions.

\section{Four reggeized gluons with a nonzero total momentum}

Since the conformal transformations do not conserve the total momentum of the gluons, to study conformal properties of the amplitudes generated by the exchange of 4 gluons in the limit $N_{c} \rightarrow \infty$ we have to generalize the derivation presented in [3] to the case when the gluons 
have their total momentum different from zero. This generalization is quite straightforward and the main change will concern the notations, which in [3] essentially used the fact that the total momentum is zero. The colour structure and the derivation lines remain the same, so that we shall be quite brief, just presenting the results.

The basic quantity is the amplitude $D_{2}$ corresponding to the exchange of two reggeized gluons (the BFKL amplitude). It satisfies the BFKL equation

$$
S_{20} D_{2}=D_{20}+g^{2} N_{c} V_{12} D_{2}
$$

where $S_{20}$ is the 2 gluon "free" Schroedinger operator for the energy $1-j$

$$
S_{20}=j-1-\omega(1)-\omega(2)
$$

$\omega(k)$ is the gluon Regge trajectory and $V_{12}$ is the BFKL interaction. We use the notation in which only the number of the gluon is indicated whose momentum enters as a variable. The inhomogeneous term for the non-forward direction and $N_{c} \rightarrow \infty$ is

$$
D_{20}(1,2)=D_{20}(2,1)=g^{2} N_{c}(f(1+2,0)-f(1,2))
$$

where $f(1,2)=f(2,1)$ is a contribution of the $q \bar{q}$ loop with gluon 1 attached to $q$ and gluon 2 attached to $\bar{q}$. Its explicit form can be easily found (see Appendix) but has no importance for the following.

The 3 gluon amplitude $D_{3}$, as in the forward case, is found to be constructed in terms of $D_{2}$ :

$$
D_{3}^{(123)}=-D_{3}^{(213)}=g \sqrt{N_{c} / 8}\left(D_{2}(2,1+3)-D_{2}(1,2+3)-D_{2}(3,1+2)\right)
$$

where the upper indeces 123 and 213 show the order of the gluons along the $q \bar{q}$ loop.

The 4-gluon amplitudes $D_{4}$ in the leading approximation in $1 / N_{c}$ correspond to neighbour gluons being in the adjoint colour state and all the gluons lying on the surface of a cylinder attached to the $q \bar{q}$ loop. There are two independent amplitudes of this type, corresponding to the order of the gluons 1234 and 2134. Both are found to be expressed via the BFKL amplitudes $D_{2}$, so that the contribution of the 4-gluon exchange reduces to a single BFKL pomeron. Explicitly one obtains in the same manner as in [3]

$$
D_{4}^{(1234)}=(1 / 4) g^{2} N_{c}\left(D_{2}(1,2+3+4)+D_{2}(4,1+2+3)-D_{2}(1+4,2+3)\right)
$$

and

$$
D_{4}^{(2134)}=(1 / 4) g^{2} N_{c}\left(D_{2}(2,1+3+4)+D_{2}(3,1+2+4)-D_{2}(1+2,3+4)-D_{2}(1+3,2+4)\right)
$$

The diffractive amplitude $D_{4}^{(0)}$, which is of main interest to us, corresponds to pairs of gluons 12 and 34 being in the vacuum colour state. It is subleading in $1 / N_{c}$ and satisfies an equation

$$
S_{40} D_{4}^{(0)}=D_{40}^{(0)}+D_{2 \rightarrow 4}^{(0)}+D_{3 \rightarrow 4}^{(0)}+D_{4 \rightarrow 4}^{(0)}+g^{2} N_{c}\left(V_{12}+V_{34}\right) D_{4}^{(0)}
$$

Here $S_{40}=j-1-\sum_{i=1}^{4} \omega(i)$,

$$
D_{40}^{(0)}=\frac{1}{2} g^{2}\left(\sum_{i=1}^{4} D_{20}(i, 1+2+3+4-i)-\sum_{i=2}^{4} D_{20}(1+i, 2+3+4-i)\right)
$$

Terms $D_{2 \rightarrow 4}^{(0)}, \ldots$ etc. come from transitions into the 4-gluon diffractive state from states with $2, \ldots$ etc gluons in the leading (cylinder) configuration. As in $[1,3]$ their sum can be presented as a certain operator $Z$ (the three-pomeron vertex) acting on the BFKL pomeron:

$$
D_{2 \rightarrow 4}^{(0)}+D_{3 \rightarrow 4}^{(0)}+D_{4 \rightarrow 4}^{(0)}=Z D_{2}
$$


¿From the explicit form of the contributions on the left-hand side, which can be found using the $s$-channel unitarity relations, one finds

$$
\begin{gathered}
Z D_{2}=(1 / 2) g^{2} \\
{[2 G(1,3+4,2)+2 G(3,1+2,4)+G(1,2+4,3)+G(1,2+3,4)+G(2,1+4,3)+G(2,1+3,4)} \\
-G(1,4,2+3)-G(1,3,2+4)-G(2,4,1+3)-G(2,3,1+4)-G(3,2,1+4)-G(3,1,2+4) \\
-G(4,2,1+3)-G(4,1,2+3)+G(2+3,0,1+4)+G(1+3,0,2+4)]
\end{gathered}
$$

In this expression function $G(1,2,3)$ is defined as the vertex $K_{2 \rightarrow 3}$ for the transition of 2 to 3 gluons, integrated with the BFKL pomeron and regularized in the infrared by terms proportional to the gluon trajectory in the same manner as in the total BFKL kernel:

$$
\begin{gathered}
G(1,2,3)=G(3,2,1)= \\
-g^{2} N_{c} W(1,2,3)-D(1,2+3)(\omega(2)-\omega(2+3))-D(1+2,3)(\omega(2)-\omega(1+2))
\end{gathered}
$$

where

$$
W(1,2,3)=\int \frac{d^{2} k_{1}^{\prime}}{(2 \pi)^{3}} K_{2 \rightarrow 3}\left(1,2,3 ; 1^{\prime}, 3^{\prime}\right) D\left(1^{\prime}, 3^{\prime}\right)
$$

and the kernel $K_{2 \rightarrow 3}$ is given by

$$
\begin{gathered}
K_{2 \rightarrow 3}\left(k_{1}, k_{2}, k_{3} ; q_{1}, q_{3}\right)= \\
-\frac{\left(k_{2}+k_{3}\right)^{2}}{\left(q_{1}-k_{1}\right)^{2} q_{3}^{2}}-\frac{\left(k_{1}+k_{2}\right)^{2}}{q_{1}^{2}\left(q_{3}-k_{3}\right)^{2}}+\frac{k_{2}^{2}}{\left(q_{1}-k_{1}\right)^{2}\left(q_{3}-k_{3}\right)^{2}}+\frac{\left(k_{1}+k_{2}+k_{3}\right)^{2}}{q_{1}^{2} q_{3}^{2}}
\end{gathered}
$$

Eq. (7) can be easily solved. Evidently the solution may be constructed as a sum of two terms corresponding to the two parts of the inhomogeneous term $D_{40}^{(0)}$ and $Z D_{2}$ :

$$
D_{4}^{(0)}=D_{4}^{D P E}+D_{4}^{T P I}
$$

The term $D_{4}^{D P E}$ coming from the inhomogeneous term $D_{40}^{(0)}$ is the DPE contribution. Its explicit form can be conveniently written using the quark loop density in the transverse coordinate space defined by the Fourier transform (see Appendix)

$$
f(1,2)=\int d^{2} r \rho_{l}(r) e^{i k_{1} r}
$$

where $l=k_{1}+k_{2}$. Then one finds

$$
D_{4}^{D P E}=(1 / 4) g^{4} N_{c} \int d^{2} r \rho_{l}(r) D_{4}^{(r)}
$$

Here $D_{4}^{(r)}$ is a convolution in the "energy" $1-j$ of two independent BFKL pomerons

$$
D_{4}^{(r)}=\int d j_{12} d j_{34} \delta\left(j-j_{12}-j_{34}\right) D_{2, j_{12}}^{(r)}(1,2) D_{2, j_{34}}^{(r)}(3,4)
$$

where the pomeron $D_{2, j}^{(r)}(1,2)$ satisfies the equation

$$
S_{20} D_{2, j}^{(r)}=\prod_{j=1}^{2}\left(e^{i k_{j} r}-1\right)+g^{2} N_{c} V_{12} D_{2, j}^{(r)}
$$

and similarly for the second pomeron. 
The part $D_{4}^{T P I}$ is the TPI contribution. It can be written as a convolution in the rapitidy space:

$$
\begin{gathered}
D_{4}^{T P I}(1,2,3,4 ; Y)= \\
\int_{0}^{Y} G_{2}\left(1,2 ; 1^{\prime} 2^{\prime} ; Y-y\right) G_{2}\left(3,4, ; 3^{\prime} 4^{\prime} ; Y-y\right) \otimes Z\left(1^{\prime}, 2^{\prime}, 3^{\prime}, 4^{\prime} ; 1^{\prime \prime}, 2^{\prime \prime}\right) \otimes D_{2}\left(1^{\prime \prime}, 2^{\prime \prime} ; y\right)
\end{gathered}
$$

where $G_{2}$ is the BFKL Green function and the symbols $\otimes$ mean integrations over intermediate momenta. This equation clearly shows that $Z$ is just the three-pomeron vertex. Its explicit form can be read from Eq. (10). As compared to the forward case studied in [3], the only difference is the appearance of a new independent argument in functions $G$.

\section{Conformal invariance}

In [2] it was shown that a vertex $V(1234)$ defined by a relation similar to (10)

$$
\begin{array}{r}
V(1234) D_{2}=\frac{1}{2} g^{2}(G(1,2+3,4)+G(2,1+3,4)+G(1,2+4,3)+G(2,1+4,3) \\
-G(1+2,3,4)-G(1+2,4,3)-G(1,2,3+4)-G(2,1,3+4)+G(1+2,0,3+4))
\end{array}
$$

is conformal invariant in the following sense. If one transforms $V D_{2}$ to the transverse coordinate space and integrates it over the 4 gluon coordinates with a conformal invariant function, the resulting integral is invariant under conformal transformation of gluon coordinates. Comparing (20) and (10) we observe that our vertex $Z$ is just a sum of permutations of gluons in $V$

$$
Z=V(1324)+V(1423)
$$

Then the conformal invariance of $Z$ trivially follows from the conformal invariance of $V$, proven in [2].

In the rest part of this section we are going to demonstrate a stronger result: not only the combination (20) of functions $G$ is conformal invariant, but each function $G(1,2,3)$ is conformal invariant by itself. So this function represents a natural generalization of the BFKL kernel not only in respect to its infrared stability but also in its conformal properties.

The proof of the conformal invariance of $G(1,2,3)$ is straightforward. The main technical problem is its transformation to the coordinate space. This task was actually already solved in [2], although there the transformation was applied to the vertex $V$ as a whole, integrated over the coordinates, which resulted in certain complications. Having this in mind, we shall present only the final expressions for $G(1,2,3)$ in the coordinate space with some comments.

Denote the integral part of $G$ in (11) as $G_{1}$ and the rest terms with the gluon trajectories as $G_{2}$. Transformation of $G_{1}$ to the coordinate space is straightforward and gives

$$
G_{1}\left(r_{1}, r_{2}, r_{3}\right)=A_{1} D_{2}\left(r_{1}, r_{3}\right)
$$

where $A_{1}$ is an operator in the coordinate space

$$
\begin{gathered}
A_{1}=\frac{g^{2} N_{c}}{8 \pi^{3}}\left[2 \pi \delta^{2}\left(r_{23}\right) \nabla_{3}^{2}\left(c-\ln r_{13}\right) \nabla_{3}^{-2}+2 \pi \delta^{2}\left(r_{12}\right) \nabla_{1}^{2}\left(c-\ln r_{13}\right) \nabla_{1}^{-2}\right. \\
\left.-2 \frac{\mathbf{r}_{12} \mathbf{r}_{23}}{r_{12}^{2} r_{23}^{2}}-2 \pi\left(c-\ln r_{13}\right)\left(\delta^{2}\left(r_{12}\right)+\delta^{2}\left(r_{23}\right)\right)-4 \pi^{2} \delta^{2}\left(r_{12}\right) \delta^{2}\left(r_{23}\right)\left(\nabla_{1}+\nabla_{3}\right)^{2} \nabla_{1}^{-2} \nabla_{3}^{-2}\right]
\end{gathered}
$$

Here $r_{12}=r_{1}-r_{2}$ etc., $c=\ln (2 / m)+\psi(1)$ where $m$ is the gluon mass acting as an infrared cutoff. The first two terms in $A_{1}$ correspond to the first two terms in (13). The last term in (23) corresponds to the last term in (13). 
The transformation of the part $G_{2}$ to the coordinate space encounters a certain difficulty in transforming the gluon Regge trajectory to the coordinate space, which requires introduction of an ultraviolet cutoff $\epsilon$. Of course the final results do not depend on $\epsilon$. One obtains

$$
G_{2}\left(r_{1}, r_{2}, r_{3}\right)=A_{2} D_{2}\left(r_{1}, r_{3}\right)
$$

where $A_{2}$ is another operator in the coordinate space

$A_{2}=-\frac{g^{2} N_{c}}{8 \pi^{3}}\left(\frac{1}{r_{23}^{2}}-2 \pi c \delta^{2}\left(r_{23}\right)\right)+\delta^{2}\left(r_{23}\right) \omega\left(-i \nabla_{3}\right)-\frac{g^{2} N_{c}}{8 \pi^{3}}\left(\frac{1}{r_{12}^{2}}-2 \pi c \delta^{2}\left(r_{12}\right)\right)+\delta^{2}\left(r_{12}\right) \omega\left(-i \nabla_{1}\right)$

The 4 terms in $A_{2}$ correspond to the 4 respective terms in $G_{2}$. The singular operators $1 / r_{12}^{2}$ and $1 / r_{23}^{2}$ are in fact defined with the help of $\epsilon$ as

$$
\frac{1}{r^{2}} \equiv \frac{1}{r^{2}+\epsilon^{2}}+2 \pi \delta^{2}(r) \ln \epsilon, \quad \epsilon \rightarrow 0
$$

They do not depend on $\epsilon$.

Summing $A_{1}$ and $A_{2}$ we find that the terms containing $\ln m$ cancel. As a result, $G\left(r_{1}, r_{2}, r_{3}\right)$ does not depend on the gluon mass and is infrared stable (which was to be expected, of course).

Now we can proceed to study the conformal invariance of the integral

$$
I=\int d^{2} r_{1} d^{2} r_{2} d^{2} r_{3} \Phi\left(r_{1}, r_{2}, r_{2}\right) G\left(r_{1}, r_{2}, r_{3}\right)
$$

where function $\Phi$ is conformal invariant. We shall demonstrate that the integral $I$ does not change under conformal transformations. In doing so we shall use the fact that the BFKL solution $\Psi\left(r_{1}, r_{2}\right)=\nabla_{1}^{-2} \nabla_{2}^{-2} D_{2}\left(r_{1}, r_{2}\right)$ is conformal invariant.

We shall study the behaviour of the function $G$ only under the inversion, the invariance under translations and rescaling being obvious. In the complex notation, under inversion

$$
r \rightarrow 1 / r, \quad k \equiv-i \partial \rightarrow r^{2} k
$$

from which we also conclude (for real $r$ )

$$
d^{2} r \rightarrow d^{2} r / r^{4}
$$

and

$$
D_{2}\left(r_{1}, r_{2}\right)=r_{1}^{4} r_{2}^{4} D_{2}\left(r_{1}, r_{2}\right)
$$

Certain parts of $G$ give contributions which are evidently invariant under inversion. Take the last term from $A_{1}$. It leads to an integral

$$
I_{1}=\frac{g^{2} N}{2 \pi} \int d^{2} r \Phi(r, r, r) \nabla^{2} \Psi(r, r)
$$

It is conformal invariant, since both functions $\Phi$ and $\Psi$ are invariant, and the factor $r^{-4}$ from $d^{2} r$ is cancelled by the factor $r^{4}$ from $\nabla^{2}$.

Terms with the denominators $r_{12}^{2}$ and/or $r_{23}^{2}$ from $A_{1}+A_{2}$ combine ito an integral

$$
I_{2}=-\frac{g^{2} N}{8 \pi^{3}} \int d^{2} r_{1} d^{2} r_{2} d^{2} r_{3} \Phi\left(r_{1}, r_{2}, r_{3}\right) \frac{r_{13}^{2}}{r_{12}^{2} r_{23}^{2}} D_{2}\left(r_{1}, r_{3}\right)
$$

in which the regularization (26) is implied. Under inversion the ultraviolet cutoff $\epsilon$ is transformed into $\epsilon_{1}=r_{1} r_{2} \epsilon$ and $\epsilon_{2}=r_{2} r_{3} \epsilon$ in the denominators $r_{12}^{2}$ and $r_{23}^{2}$ respectively. This gives rise to a change of $I_{2}$ under inversion:

$$
\Delta I_{2}=\frac{g^{2} N}{4 \pi^{2}} \int d^{2} r_{1} d^{2} r_{3}\left(\Phi\left(r_{1}, r_{1}, r_{3}\right) \ln r_{1}^{2}+\Phi\left(r_{1}, r_{3}, r_{3}\right) \ln r_{3}^{2}\right) D_{2}\left(r_{1}, r_{3}\right)
$$


The rest of the terms in $A_{1}+A_{2}$, proportional either to $\delta^{2}\left(r_{12}\right)$ or to $\delta^{2}\left(r_{23}\right)$ can be divided into two parts. The first contains terms in which the $\delta$-function is multiplied either by a constant or by $\ln r_{13}$ It gives rise to a part of the integral $I_{3}$. Terms with a constant are evidently invariant under inversion. However those containing $\ln r_{13}$ are not and the corresponding change of $I_{3}$ is trivially found to be

$$
\Delta I_{3}=-\frac{g^{2} N}{8 \pi^{2}} \int d^{2} r_{1} d^{2} r_{3}\left(\Phi\left(r_{1}, r_{3}, r_{3}\right)+\Phi\left(r_{1}, r_{1}, r_{3}\right)\right) \ln \left(r_{1}^{2} r_{3}^{2}\right) D_{2}\left(r_{1}, r_{3}\right)
$$

The second part contains differential operators acting on $D_{2}$. It has a form

$$
-\frac{g^{2} N_{c}}{8 \pi^{2}}\left(a_{1} \delta^{2}\left(r_{12}\right)+a_{3} \delta^{2}\left(r_{23}\right)\right)
$$

where

$$
a_{1}=\nabla_{1}^{2} \ln r_{13}^{2} \nabla_{1}^{-2}+\ln \left(-\nabla_{1}^{2}\right) \equiv \nabla_{1}^{2} \tilde{a}_{1} \nabla_{1}^{-2}
$$

and $a_{3}$ is obtained by interchange $1 \leftrightarrow 3$. The operator $a_{1}$ can be transformed into a different form in which its properties under inversion become apparent. Indeed we have, in the complex notation

$$
\tilde{a}_{1}=\ln r_{13}+\ln k_{1}+c . c
$$

One can prove $[6]$ that

$$
\ln r_{13}+\ln k_{1}=\ln \left(r_{13}^{2} k_{1}\right)-k_{1}^{-1} \ln r_{13} k_{1}
$$

from which one finds

$$
a_{1}=k_{1} \ln \left(r_{13}^{2} k_{1}\right) k_{1}^{-1}-\ln r_{13}+c . c
$$

Under the inversion

$$
\ln r_{13}^{2} k_{1} \rightarrow \ln r_{13}^{2} k_{1}-2 \ln r_{3}
$$

so that the change in $a_{1}$ is

$$
\Delta a_{1}=-2 \ln r_{13}+\ln \left(r_{1} r_{3}\right)+\text { c.c. }=\ln \frac{r_{1}^{2}}{r_{3}^{2}}
$$

It follows that the change of the last part of the integral $I_{4}$, which comes from (35), is

$$
\Delta I_{4}=-\frac{g^{2} N}{8 \pi^{2}} \int d^{2} r_{1} d^{2} r_{3}\left(\Phi\left(r_{1}, r_{1}, r_{3}\right)-\Phi\left(r_{1}, r_{3}, r_{3}\right)\right) D_{2}\left(r_{1}, r_{3}\right) \ln \frac{r_{1}^{2}}{r_{3}^{2}}
$$

In the sum all the changes cancel: $\Delta I_{2}+\Delta I_{3}+\Delta I_{4}=0$, which proves that the total integral $I$ is indeed invariant under inversion.

\section{Double pomeron exchange vs. triple pomeron}

Let us return to the 4-gluon equation for the diffractive amplitude

$$
S_{40} D_{4}^{(0)}=D_{40}^{(0)}+Z D_{2}+g^{2} N_{c}\left(V_{12}+V_{34}\right) D_{4}^{(0)}
$$

The solution of this equation is a known function, constructed as a sum of the DPE and a TPI parts as in Eq. (14). Each part consists of several terms, corresponding to various terms in $D_{40}^{(0)}$ and $Z D_{2}$ (see Eqs. (8) and (10)). 
Let us separate from this known exact solution some arbitrary function $f$, which may depend on the angular momentum $j$ :

$$
D_{4}^{(0)}(j)=f(j)+\tilde{D}_{4}^{(0)}(j)
$$

Putting this into (43) we obtain an equation for the new 4-gluon function $\tilde{D}_{4}^{(0)}$

$$
S_{40} \tilde{D}_{4}^{(0)}=D_{40}^{(0)}+Z D_{2}-\left(S_{40}-g^{2} N_{c}\left(V_{12}+V_{34}\right)\right) f+g^{2} N_{c}\left(V_{12}+V_{34}\right) \tilde{D}_{4}^{(0)}
$$

So the inhomogeneous term has changed

$$
D_{40}^{(0)}+Z D_{2} \rightarrow D_{40}^{(0)}+Z D_{2}-\left(S_{40}-g^{2} N_{c}\left(V_{12}+V_{34}\right)\right) f
$$

The significance of this seemingly trivial procedure is that if one chooses $f$ to be the BFKL function, depending on some gluon momenta, the added term aquires a structure of the triple pomeron contribution. So the net effect of this procedure will be to add some new triple pomeron term and simultaneously to add a new simpler term $f$ to the amplitude itself. This means that one can calculate some specific triple pomeron contributions expressing them in terms of simple functions.

Let us see how this procedure works in some important cases. We shall consider only the forward case, for simplicity. Let $f=(1 / 2) g^{2} D_{2}(1)=(1 / 2) g^{2} D_{2}(2+3+4)$. We are going to calculate then

$$
X=(1 / 2) g^{2}\left(S_{40}-g^{2} N_{c}\left(V_{12}+V_{34}\right)\right) D_{2}(1)
$$

Using Eq. (1) we can express the $j-1$ term in $S_{40}$ in terms of the forward BFKL interaction. $V_{0}$ and $\omega$ to obtain

$$
X=(1 / 2) g^{2} D_{20}+(1 / 2) g^{2}\left(g^{2} N_{c}\left(V_{0}-V_{12}-V_{34}\right)+2 \omega(1)-\sum \omega(i)\right) D_{2}(1)
$$

It is straightforward to find that

$$
\left(V_{0}-V_{12}\right) D_{2}(1)=-W(1,2,3+4)
$$

where $W$ has been defined by (12). The bootstrap relation gives

$$
g^{2} N_{c} V_{34} D_{2}(1)=2(\omega(3+4)-\omega(3)-\omega(4)) D_{2}(1)
$$

Passing to functions $G(1,3)$ with two arguments defined as $G(1,2,3)$, Eq. (11), for $1+2+3=0$ we finally obtain

$X=(1 / 2) g^{2}\left(D_{20}(1)+G(1,3+4)+D_{2}(1)(\omega(3)+\omega(4)-2 \omega(3+4))+D_{2}(1+2)(\omega(2)-\omega(1+2))\right.$

If we put this into equation (43), we find that the changed function $\tilde{D}_{4}^{(0)}$ will satisfy it with a new inhomogeneous term

$$
\begin{gathered}
D_{40}+Z D_{2}+(1 / 2) g^{2}\left(-D_{20}(1)-G(1,3+4)\right. \\
-D_{2}(1)(\omega(3)+\omega(4)-2 \omega(3+4))-D_{2}(1+2)(\omega(2)-\omega(1+2))
\end{gathered}
$$

Note that the additional term $-(1 / 2) g^{2} D_{20}$ will cancel the identical term in $D_{40}^{(0)}$. As a result, we have converted the double pomeron exchange contribution coming from $(1 / 2) g^{2} D_{20}(1)$ into a triple pomeron contribution corresponding essentially to $G(1,3+4)$ plus the explicitly separated term $(1 / 2) g^{2} D_{2}(1)$. In other words, one can calculate the triple pomeron contribution corresponding to a vertex

$$
(1 / 2) g^{2}\left(-G(1,3+4)-D_{2}(1)(\omega(3)+\omega(4)-2 \omega(3+4))-D_{2}(1+2)(\omega(2)-\omega(1+2))\right.
$$


as a sum of the double pomeron exchange coupled to $-(1 / 2) g^{2} D_{20}(1)$ and a term $(1 / 2) g^{2} D_{2}(1)$.

Evidently this result is trivially generalized for $f=D_{2}(i), i=2,3,4$ by simple permutation of indeces $1,2,3$ and 4 .

Now let us consider a case when $f=(1 / 2) g^{2} D_{2}(1+2)$. In this case

$$
X=(1 / 2) g^{2} D_{20}+(1 / 2) g^{2}\left(g^{2} N_{c}\left(V_{0}-V_{12}-V_{34}\right)+2 \omega(1+2)-\sum \omega(i)\right) D_{2}(1+2)
$$

In terms of $W$ we have

$$
V_{0} D_{2}(1+2)=-W(1+2,0,3+4)
$$

The bootstrap gives

$$
g^{2} N_{c}\left(V_{12}+V_{34}\right) D_{2}(1+2)=2 D_{2}(1+2)\left(2 \omega(1+2)-\sum \omega(i)\right)
$$

so that in terms of $G$ we obtain

$$
X=(1 / 2) g^{2}\left(-D_{20}(1+2)+G(1+2,3+4)-D(1+2)\left(4 \omega(1+2)-\sum \omega(i)\right)\right)
$$

Again we see that the term with the double pomeron exchange coupled to $g^{2}(1 / 2) D_{20}(1+2)$ can be transformed into a triple pomeron vertex, essentially, into $-G(1+2,3+4)$ term.

Finally we study a more complicated case with $f=(1 / 2) g^{2} D_{2}(1+3)$. In this case we find

$$
V_{0} D_{2}(1+3)=-W(1+3,0,2+4)
$$

Calculation of $V_{12}$ or $V_{34}$ applied to $D_{2}(1+3)$ is done using the formula derived in the appendix to [3]. It gives

$$
V_{12} D_{2}(1+3)=W(2+4,1,3)+W(4,2,1+3)-W(3,1+2,4)-W(1+3,0,2+4)
$$

and

$$
V_{34} D_{2}(1+3)=W(2+4,3,1)+W(2,4,1+3)-W(1,3+4,2)-W(1+3,0,2+4)
$$

Using these results we obtain for this case

$$
\begin{gathered}
X=(1 / 2) g^{2}\left(D_{20}(1+3)+G(1,2+4)+G(2,1+3)+G(3,2+4)+G(4,1+3)\right) \\
-G(1,2)-G(3,4)-G(1+3,2+4)-D_{2}(1)(\omega(3+4)-\omega(3)) \\
\left.-D_{2}(2)(\omega(3+4)-\omega(4))-D_{2}(3)(\omega(1+2)-\omega(1))-D_{2}(4)(\omega(1+2)-\omega(2))\right)
\end{gathered}
$$

The result for $f=(1 / 2) g^{2} D_{2}(1+4)$ is obtained from this after the permutation of 3 and 4 .

Inspecting these results and comparing them with the form of our triple pomeron vertex, we see that only four terms

$$
(1 / 2) g^{2}(G(1,3)+G(1,4)+G(2,3)+G(2,4))
$$

are not changed under these transformations and thus correspond to a true triple pomeron interaction. All the rest can be transformed into terms which are essentially double pomeron exchange contribution. Conversely, one can eliminate terms from the double pomeron exchange substituting them by equivalent triple pomeron contributions.

The most radical result follows if one takes

$$
f=D_{40}^{(0)}\left(D_{20} \rightarrow D_{2}\right)
$$


In this case all the double exchange becomes cancelled and the whole amplitude is given by a sum of two terms (in an evident symbolic notation)

$$
D_{4}=D_{40}\left(D_{20} \rightarrow D_{2}\right)+\int_{0}^{Y} G_{2}(Y-y) G_{2}(Y-y) \tilde{Z} D_{2}(y)
$$

where the new vertex is found to be

$$
\begin{aligned}
\tilde{Z} D_{2}= & (1 / 2) g^{2}(G(1,3)+G(1,4)+G(2,3)+G(2,4)+G(1+2,3+4) \\
& -G(1,3+4)-G(2,3+4)-G(3,1+2)-G(4,1+2))
\end{aligned}
$$

Comparing to (20) for the forward case, we observe that it coincides with the part $V(1234)$ of the vertex introduced in [1].

\section{Coupling to pomerons. Comparison with the dipole ap- proach}

Using the possibility to transfer the DPE part into the TPI one, we shall study the triple pomeron vertex in the simpler form (20) for a non-forward direction. In the coordinate space of 4 gluons, the dependence on only the sum of the momenta of two gluons, say, $1+2$, is translated into a factor $\delta^{2}\left(r_{12}\right)$, so that the two gluons have to be taken at the same point. However the wave functions $\Psi\left(r_{1}, r_{2}\right)$ and $\Psi\left(r_{3}, r_{4}\right)$ of the two final pomerons coupled to the vertex vanish if $r_{1}=r_{2}$ and $r_{3}=r_{4}$ respectively. Therefore all terms in Eq.(20) which depend either only on the sum $1+2$ or/and only on the sum $3+4$ give zero, coupled to the two pomerons. This leaves only the four terms, corresponding to the mentioned "true" triple pomeron vertex

$$
\tilde{Z} D_{2}=(1 / 2) g^{2}(G(1,2+4,3)+G(1,2+3,4)+G(2,1+4,3)+G(2,1+3,4))
$$

Both pomeron functions $\Psi\left(r_{1}, r_{2}\right)$ and $\Psi\left(r_{3}, r_{4}\right)$ are symmetric in their respective arguments, due to the positive signature of the pomeron. Therefore all terms in (65) give identical contributions so that we can take

$$
\tilde{Z} D_{2}=2 g^{2} G(1,2+3,4)
$$

Turning to the explicit expression of $G(1,2,3)$ in the coordinate space, found in Sec. 3, we can split it into a "proper part"

$$
G^{p r}\left(r_{1}, r_{2}, r_{3}\right)=-\frac{g^{2} N_{c}}{8 \pi^{3}} \frac{r_{13}^{2}}{r_{12}^{2} r_{23}^{2}} D_{2}\left(r_{1}, r_{3}\right)
$$

(with the regularization (26) implied) and an "improper part" including all the rest terms, proportional to $\delta^{2}\left(r_{12}\right)$ or/and $\delta^{2}\left(r_{23}\right)$. Noting that in the coordinate space (66) is proportional to $\delta^{2}\left(r_{23}\right)$, we find that in the improper part at least three gluons, either 123 or 234, are to be taken at the same point in the transverse space. Then these terms will vanish due to the mentioned property of the pomeron wave function. Therefore the final triple pomeron vertex is given by only the proper part of $G$, Eq. (67).

Coupling this triple pomeron vertex to the two final pomerons, we arrive at the following expression for the triple pomeron contribution to the diffractive (non-forward) amplitude:

$$
D_{4}^{T P I}(Y)=-\frac{g^{4} N}{4 \pi^{3}} \int_{0}^{Y} d y \int d^{2} r_{1} d^{2} r_{2} d^{2} r_{3} \frac{r_{13}^{2}}{r_{12}^{2} r_{23}^{2}} D_{2}\left(r_{1}, r_{3} ; y\right) \Psi_{1}\left(r_{1}, r_{2} ; Y-y\right) \Psi_{2}\left(r_{2}, r_{3} ; Y-y\right)
$$


Expressing the initial pomeron via the non-amputated function $\Psi$ we can rewrite (68) as

$D_{4}^{T P I}(Y)=-\frac{g^{4} N}{4 \pi^{3}} \int_{0}^{Y} d y \int \frac{d^{2} r_{1} d^{2} r_{2} d^{2} r_{3}}{r_{13}^{2} r_{12}^{2} r_{23}^{2}} \Psi_{1}\left(r_{1}, r_{2} ; Y-y\right) \Psi_{2}\left(r_{2}, r_{3} ; Y-y\right) r_{13}^{4} \nabla_{1}^{2} \nabla_{3}^{2} \Psi\left(r_{1}, r_{3} ; y\right)$

In this form it is evident that the triple pomeron vertex is not symmetric with respect to the initial pomeron and two final ones: there appears an extra operator $r_{13}^{4} \nabla_{1}^{2} \nabla_{3}^{2}$ acting on the initial pomeron. Note that this operator is essentially a product of the Kasimir operators of the conformal group for the holomorphic and antiholomorphic parts:

$$
M^{2}(1,3) \bar{M}^{2}(1,3)=(1 / 16) r_{13}^{4} \nabla_{1}^{2} \nabla_{3}^{2}
$$

So one expects simplifications to occur provided one passes to the conformal basis for the BFKL solutions.

This basis is formed by functions (in complex notation)

$$
E_{n, \nu, r_{0}}\left(r_{1}, r_{2}\right) \equiv E_{\mu}\left(r_{1}, r_{2}\right)=\left(\frac{r_{12}}{r_{10} r_{20}}\right)^{\frac{1-n}{2}+i \nu}\left(\frac{r_{12}^{*}}{r_{10}^{*} r_{20}^{*}}\right)^{\frac{1+n}{2}+i \nu}
$$

They are proper functions of (70) In fact,

$$
M^{2} \bar{M}^{2} E_{\mu}\left(r_{1}, r_{2}\right)=\frac{\pi^{8}}{4} \frac{E_{\mu}\left(r_{1}, r_{2}\right)}{a_{n+1, \nu} a_{n-1, \nu}}
$$

where we use the standard notation

$$
a_{n, \nu} \equiv a_{\mu}=\frac{\pi^{4}}{2} \frac{1}{\nu^{2}+n^{2} / 4}
$$

They form a complete system:

$$
r_{12}^{4} \delta^{2}\left(r_{11^{\prime}}\right) \delta^{2}\left(r_{22^{\prime}}\right)=\sum_{\mu} E_{\mu}\left(r_{1}, r_{2}\right) E_{\mu}^{*}\left(r_{1}^{\prime}, r_{2}^{\prime}\right)
$$

where we use a notation

$$
\sum_{\mu}=\sum_{n=-\infty}^{\infty} \int d \nu \frac{1}{2 a_{n, \nu}} \int d^{2} r_{0}
$$

and satisfy the orthogonality relation

$$
\begin{gathered}
\int \frac{d^{2} r_{1} d^{2} r_{2}}{r_{12}^{4}} E_{n, \nu, r_{0}}\left(r_{1}, r_{2}\right) E_{n^{\prime}, \nu^{\prime}, r_{0}^{\prime}}^{*}\left(r_{1}, r_{2}\right) \\
=a_{n, \nu} \delta_{n n^{\prime}} \delta\left(\nu-\nu^{\prime}\right) \delta^{2}\left(r_{00^{\prime}}\right)+b_{n \nu} \delta_{n,-n^{\prime}} \delta\left(\nu+\nu^{\prime}\right)\left|r_{00^{\prime}}\right|^{-2-4 i \nu}\left(\frac{r_{00^{\prime}}}{r_{00^{\prime}}^{*}}\right)^{n}
\end{gathered}
$$

The coefficients $b_{n \nu}$ may be found in [7]. Using these properties one can express the pomeron wave function as

$$
\Psi\left(r_{1}, r_{2} ; y\right)=\sum_{\mu} e^{y \omega_{\mu}} E_{\mu}\left(r_{1}, r_{2}\right)\left\langle\mu \mid \Psi_{0}\right\rangle
$$

where $\omega_{\mu}=\omega_{n \nu}$ are the eigenvalues of the BFKL kernel and we have defined

$$
\left\langle\mu \mid \Psi_{0}\right\rangle=\int \frac{d^{2} r_{1} d^{2} r_{2}}{r_{12}^{4}} E_{\mu}^{*}\left(r_{1}, r_{2}\right) \Psi_{0}\left(r_{1}, r_{2}\right)
$$


We present all the three pomerons in (69) as a superposition (77) of conformal states. As mentioned, the operator acting on the initial pomeron is essentially a product of Kasimir operators, so we can use Eq. (70). We then obtain, after the integration over $y$

$$
\begin{gathered}
D_{4}^{T P I}(Y)=-\frac{g^{4} N}{4 \pi^{3}} \sum_{\mu, \mu_{1}, \mu_{2}}\left\langle\mu \mid \Psi_{0}\right\rangle\left\langle\mu_{1} \mid \Psi_{10}\right\rangle\left\langle\mu_{2} \mid \Psi_{20}\right\rangle \frac{e^{Y\left(\omega_{\mu_{1}}+\omega_{\mu_{2}}\right)}-e^{Y \omega_{\mu}}}{\omega_{\mu_{1}}+\omega_{\mu_{2}}-\omega_{\mu}} \\
\frac{4 \pi^{8}}{a_{n-1, \nu} a_{n+1, \nu}} \int \frac{d^{2} r_{1} d^{2} r_{2} d^{2} r_{3}}{r_{13}^{2} r_{12}^{2} r_{23}^{2}} E_{\mu_{1}}\left(r_{1}, r_{2}\right) E_{\mu_{2}}\left(r_{2}, r_{3}\right) E_{\mu}\left(r_{1}, r_{3}\right)
\end{gathered}
$$

In this form the triple pomeron contribution can be compared to the double dipole density found by Peschanski [4] in A.H.Mueller's colour dipole approach. One observes that the two expressions differ only in the sign and factor

$$
\frac{4 \pi^{8}}{a_{n-1, \nu} a_{n+1, \nu}}
$$

which in our approach distinguishes the initial pomeron from the two final ones. The integral over the coordinates of the three pomerons is the same. So essentially the three-pomeron contribution to the diffractive amplitude found in our approach coincides with the double dipole density in the dipole approach.

However one should not forget that in our $s$-channel unitarity approach the TPI term (79) does not exhaust all the diffractive amplitude. From the derivation of Sec. 4 it follows

$$
D_{4}^{(0)}=D_{40}\left(D_{20} \rightarrow D_{2}\right)+D_{4}^{T P I}
$$

At high energies the TPI term behaves essentially as $s^{2 \Delta}$ and the first one as $s^{\Delta}$ where $\Delta$ is the BFKL intercept. So one might think that the first term could be neglected. However the correct region of the validity of the leading log approximation, implicit in the hard pomeron theory, is $g^{2} \ln s \sim 1$ when the two terms in (80) have the same order of magnitude. The dipole approach uses essentially the same leading log approximation. Therefore the fact that it leads to the double dipole density which coincides only with the TPI term in the $s$-channel unitarity approach and shows no trace of the first term points to certain differences between the two approaches.

\section{Higher-order densities in the dipole approach}

In the colour dipole formalism the $\mathrm{k}$-fold inclusive dipole density is obtained as the $k$-th functional derivative of the functional $D\left\{u\left(r_{i}, r_{f}\right)\right\}$, taken at $u\left(r_{i}, r_{f}\right)=1$ [8]. The arguments $r_{i}$ and $r_{f}$ are the dipole endpoints in the transverse plane. In the following in many cases we denote them as a single variable $r$ for brevity. The functional $D$ satisfies a simple equation

$$
\begin{gathered}
D\left(r_{1}, r_{0}, y, u\right)=u\left(r_{1}, r_{0}\right) e^{2 y \omega\left(r_{10}\right)} \\
+\frac{g^{2} N_{c}}{8 \pi^{3}} \int_{0}^{y} d y^{\prime} e^{2\left(y-y^{\prime}\right) \omega\left(r_{10}\right)} \int d^{2} r_{2} \frac{r_{10}^{2}}{r_{12}^{2} r_{20}^{2}} D\left(r_{1}, r_{2}, y^{\prime}, u\right) D\left(r_{2}, r_{0}, y^{\prime}, u\right)
\end{gathered}
$$

Here $r_{1}$ and $r_{0}$ are the end points of the $q \bar{q}$ pair which determine the initial dipole; $\omega(r)$ is not a Fourier transform of the trajectory, but just $\omega(k)$ with $k / m$ formally substituted by $r / \epsilon$, where $\epsilon$ is an ultraviolet cutoff. This cutoff is also implied in the singular kernel of the integral operator in $r_{2}$. Eq. (81) is compatible with the normalization condition $D(u=1)=1$. 
Taking the $k$-th derivative we arrive at an equation for the $k$-fold dipole density. For $k>1$ we obtain

$$
\begin{gathered}
n_{k}\left(r_{1}, r_{0} ; \rho_{1}, \ldots \rho_{k} ; y\right)=\frac{g^{2} N_{c}}{8 \pi^{3}} \int_{0}^{y} d y^{\prime} e^{2\left(y-y^{\prime}\right) \omega\left(r_{10}\right)} \int d^{2} r_{2} \frac{r_{10}^{2}}{r_{12}^{2} r_{20}^{2}} n_{k}\left(r_{1}, r_{2} ; \rho_{1}, \ldots \rho_{k} ; y^{\prime}\right)+\left(r_{1} \leftrightarrow r_{0}\right) \\
+\frac{g^{2} N_{c}}{8 \pi^{3}} \int_{0}^{y} d y^{\prime} e^{2\left(y-y^{\prime}\right) \omega\left(r_{10}\right)} \int d^{2} r_{2} \frac{r_{10}^{2}}{r_{12}^{2} r_{20}^{2}} \sum_{l=1}^{k-1}\left(n_{l}\left(r_{1}, r_{2} ; \rho_{1}, \ldots \rho_{l} ; y^{\prime}\right) n_{k-l}\left(r_{2}, r_{0} ; \rho_{l+1}, \ldots \rho_{k} ; y^{\prime}\right)\right. \\
+ \text { symmetrization terms })
\end{gathered}
$$

where the symmetrization terms (ST) are obtained from the explicitly shown one by taking all different divisions of arguments $\rho_{1}, \ldots \rho_{k}$ into two groups with $l$ and $l-k$ arguments. For $k=1$ an inhomogeneous term should be added whose form is clear from (81). One should note that the operator on the right-hand side acts nontrivially only on the first argument of the density $n_{k}$. Its action on the rapidity variable, on the contrary, is rather simple. Multiplying the equation by $e^{-2 y \omega\left(r_{10}\right)}$, differentiating then with respect to $y$ and passing to the $j$-space by the standard Mellin transformation one obtains

$$
\begin{gathered}
(j-1) n_{k}\left(r_{1}, r_{0} ; \rho_{1}, \ldots \rho_{k} ; j\right)=\frac{g^{2} N_{c}}{4 \pi^{3}} \int d^{2} r_{2} L\left(r_{1}, r_{2}, r_{20}\right) n_{k}\left(r_{1}, r_{2} ; \rho_{1}, \ldots \rho_{k} ; y\right) \\
+\frac{g^{2} N_{c}}{8 \pi^{3}} \int \frac{d j_{1} d j_{2}}{(2 \pi i)^{2}\left(j+1-j_{1}-j_{2}\right)} \int d^{2} r_{2} \frac{r_{10}^{2}}{r_{12}^{2} r_{20}^{2}} \\
\sum_{l=1}^{k-1}\left(n_{l}\left(r_{1}, r_{2} ; \rho_{1}, \ldots \rho_{l} ; j_{1}\right) n_{k-l}\left(r_{2}, r_{0} ; \rho_{l+1}, \ldots \rho_{k} ; j_{2}\right)+S T\right)
\end{gathered}
$$

where we introduced the BFKL kernel in the coordinate space

$$
L\left(r_{12}, r_{20}\right)=\frac{r_{10}^{2}}{\left(r_{12}^{2}+\epsilon^{2}\right)\left(r_{20}^{2}+\epsilon^{2}\right)}-2 \pi \ln \frac{r_{10}}{\epsilon}\left(\delta^{2}\left(r_{12}\right)+\delta^{2}\left(r_{20}\right)\right.
$$

Comparing with (26) we see that it does not depend on $\epsilon$ and is ultraviolet stable.

To solve this equation we present the dependence of the densities on their first two arguments in the conformal basis:

$$
n_{k}\left(r_{1}, r_{0}\right)=\sum_{\mu} E_{\mu}\left(r_{1}, r_{0}\right) n_{k}^{\mu}
$$

Here we have suppressed all other arguments in $n_{k}$ irrelevant for the time being. The densities $n_{k}^{\mu}$ in a given conformal state are obtained from $n_{k}\left(r_{1}, r_{0}\right)$ by the inverse transformation which follows from property (76) and a relation between $E_{n, \nu}$ and $E_{-n,-\nu}$ (see [7])

$$
n_{k}^{\mu}=\int \frac{d^{2} r_{1} d^{2} r_{0}}{r_{10}^{4}} E_{\mu}^{*}\left(r_{1}, r_{0}\right) n_{k}\left(r_{1}, r_{0}\right)
$$

So, to pass to the conformal basis, we integrate Eq. (83) over $r_{1}$ and $r_{0}$ as indicated in (86). The first term on the right-hand side can be simplified due to the property of the BFKL kernel

$$
\frac{g^{2} N_{c}}{4 \pi^{3}} \int d^{2} r_{2} L\left(r_{12}, r_{20}\right) E_{\mu}\left(r_{1}, r_{2}\right)=\omega_{\mu} E_{\mu}\left(r_{1}, r_{0}\right)
$$

Therefore after the integration we obtain

$$
\left(j-1-\omega_{\mu}\right) n_{k}^{\mu}\left(\rho_{1}, \ldots \rho_{k}, j\right)=\frac{g^{2} N_{c}}{8 \pi^{3}} \int \frac{d j_{1} d j_{2}}{(2 \pi i)^{2}\left(j+1-j_{1}-j_{2}\right)}
$$




$$
\int \frac{d^{2} r_{1} d^{2} r_{2} d^{2} r_{0}}{r_{12}^{2} r_{20}^{2} r_{10}^{2}} E_{\mu}^{*}\left(r_{10}\right) \sum_{l=1}^{k-1}\left(n_{l}\left(r_{1}, r_{2} ; \rho_{1}, \ldots \rho_{l} ; j_{1}\right) n_{k-l}\left(r_{2}, r_{0} ; \rho_{l+1}, \ldots \rho_{k} ; j_{2}\right)+S T\right)
$$

To find the final form of the equation we have only to present also the densities $n_{l}$ and $n_{k-l}$ as functions of their first arguments in the form (86). Then we get

$$
\begin{gathered}
\left(j-1-\omega_{\mu}\right) n_{k}^{\mu}\left(\rho_{1}, \ldots \rho_{k}, j\right)=\int \frac{d j_{1} d j_{2}}{(2 \pi i)^{2}\left(j+1-j_{1}-j_{2}\right)} \\
\sum_{\mu_{1}, \mu_{2}} V_{\mu, \mu_{1} \mu_{2}} \sum_{l=1}^{k-1}\left(n_{l}^{\mu_{1}}\left(\rho_{1}, \ldots \rho_{l} ; j_{1}\right) n_{k-l}^{\mu_{2}}\left(\rho_{l+1}, \ldots \rho_{k} ; j_{2}\right)+S T\right)
\end{gathered}
$$

where

$$
V_{\mu \mu_{1} \mu_{2}}=\frac{g^{2} N_{c}}{8 \pi^{3}} \int \frac{d^{2} r_{1} d^{2} r_{2} d^{2} r_{0}}{r_{12}^{2} r_{20}^{2} r_{10}^{2}} E_{\mu}^{*}\left(r_{10}\right) E_{\mu_{1}}\left(r_{12}\right) E_{\mu_{2}}\left(r_{20}\right)
$$

is just one half of the three-pomeron vertex introduced by Peschanski.

Eq. (89) allows to obtain succesively dipole densities for any number of dipoles starting from the lowest order one-dipole density, for which

$$
n_{1}^{\mu}(\rho)=\frac{E_{\mu}^{*}(\rho)}{j-1-\omega_{\mu}} \frac{1}{\rho^{4}}
$$

(we recall that in this notation $\rho$ includes two endpoints of the dipole $\rho_{i}$ and $\rho_{f} ; \rho^{2} \equiv \rho_{i f}^{2}$ ).

Putting this into (89) for $k=2$ and integrating over $j_{1}$ and $j_{2}$ we arrive at the expression obtained by Peschanski

$$
n_{2}^{\mu}\left(\rho_{1}, \rho_{2} ; j\right)=\frac{1}{\omega-\omega_{\mu}} \sum_{\mu_{1}, \mu_{2}} \frac{1}{\omega-\omega_{\mu_{1}}-\omega_{\mu_{2}}} V_{\mu, \mu_{1}, \mu_{2}} E_{\mu_{1}}^{*}\left(\rho_{1}\right) E_{\mu_{2}}^{*}\left(\rho_{2}\right) \frac{1}{\rho_{1}^{4} \rho_{2}^{4}}
$$

where $\omega=j-1$. (To compare with [5] one should take into acount that factors $1 /\left(2 a_{\mu}\right)$ are included in the definition of sums over $\mu$ 's in our notation).

Now we continue this process and study the density for three dipoles. Eq. (89) for $k=3$ reads

$$
\begin{gathered}
n_{3}^{\mu}\left(\rho_{1}, \rho_{2}, \rho_{3} ; j\right)=\frac{1}{\omega-\omega_{\mu}} \int \frac{d j_{1} d j_{2}}{(2 \pi i)^{2}\left(j+1-j_{1}-j_{2}\right)} \\
\sum_{\mu_{1}, \mu_{2}} V_{\mu, \mu_{1} \mu_{2}}\left(n_{1}^{\mu_{1}}\left(\rho_{1} ; j_{1}\right) n_{2}^{\mu_{2}}\left(\rho_{2}, \rho_{3} ; j_{2}\right)+S T\right)
\end{gathered}
$$

Let us study the term written explicitly. We put in it the expressions for $n_{1}^{\mu_{1}}$ and $n_{2}^{\mu_{2}}$ obtained earlier. Then we get, after integrations over $j_{1}$ and $j_{2}$ :

$$
\begin{gathered}
n_{3}^{\mu}\left(\rho_{1}, \rho_{2}, \rho_{3} ; j\right)=\frac{1}{\omega-\omega_{\mu}} \sum_{\mu_{1}, \mu_{2}, \mu_{3}, \mu_{4}} V_{\mu, \mu_{1} \mu_{2}} V_{\mu_{2} \mu_{3} \mu_{4}} \\
\frac{1}{\left(\omega-\omega_{\mu_{1}}-\omega_{\mu_{2}}\right)\left(\omega-\omega_{\mu_{1}}-\omega_{\mu_{3}}-\omega_{\mu_{4}}\right)} E_{\mu_{1}}^{*}\left(\rho_{1}\right) E_{\mu_{3}}^{*}\left(\rho_{2}\right) E_{\mu_{4}}^{*}\left(\rho_{3}\right) \frac{1}{\rho_{1}^{4} \rho_{2}^{4} \rho_{3}^{4}}
\end{gathered}
$$

To this term we have to add terms which symmetrize in the three dipoles.

Studying (94) we see that it corresponds to the picture when first the initial pomeron splits into two pomerons, 1 and 2, and afterwards the pomeron 2 splits into pomerons 3 and 4. One does not find here a local vertex for the transition of the initial pomeron into three final ones. It is not difficult to see under which condition one would get such a local vertex. 
If we forget about the dependence of the second denominator on $\mu_{2}$, then one can sum over $\mu_{2}$. Using (74) one obtains

$$
\sum_{\mu_{2}} V_{\mu \mu_{1} \mu_{2}} V_{\mu_{2} \mu_{3} \mu_{4}}=\left(\frac{g^{2} N_{c}}{8 \pi^{3}}\right)^{2} \int \frac{d^{2} r_{1} d^{2} r_{2} d^{2} r_{3} d^{2} r_{0}}{r_{12}^{2} r_{10}^{2} r_{23}^{2} r_{30}^{2}} E_{\mu}^{*}\left(r_{1}, r_{0}\right) E_{\mu_{1}}\left(r_{1}, r_{2}\right) E_{\mu_{3}}\left(r_{2}, r_{3}\right) E_{\mu_{4}}\left(r_{3}, r_{0}\right)
$$

which is just the vertex from one to three pomerons introduced by Peschanski in [5]. However, the described summation is not possible due to the second denominator. It implies that the pomeron 2 has to evolve in $y$ from the point of its formation from the initial pomeron up to the point of its splitting into the final pomerons 3 and 4 .

Thus our conclusion is that the vertex for transition from 1 to $k$ pomerons introduced by Peschanski, in fact, does not appear in the solution of the Mueller equation for the $k$-fold density, which rather corresponds to a set of all fan diagrams with only the triple pomeron coupling. Absence of higher-order couplings can be directly traced to the structure of the equation (81) for the generating functional $D$, quadratic in $D$.

To conclude this section we note that at asymptotic energies the higher- order densities in the dipole approach correspond to the standard Regge-Gribov picture, in the tree approximation (fan diagrams), with only the triple pomeron interaction, which however has a highly complicated non-local form. Indeed, the triple pomeron interaction present in (94) corresponds to a structure

$$
T=\frac{g^{2} N_{c}}{8 \pi^{3}} \int \frac{d^{2} r_{1} d^{2} r_{2} d^{2} r_{3}}{r_{12}^{2} r_{23}^{2} r_{31}^{2}} \tilde{G}_{3}\left(r_{1}, r_{2} ; r_{1}^{\prime}, r_{2}^{\prime}\right) \tilde{G}_{1}\left(r_{2}, r_{3} ; r_{2}^{\prime}, r_{3}^{\prime}\right) \tilde{G}_{2}\left(r_{3}, r_{1} ; r_{3}^{\prime}, r_{1}^{\prime}\right)
$$

where $\tilde{G}_{i}, i=1,2,3$ are Green functions of the interacting pomerons defined as

$$
\tilde{G}\left(r_{1}, r_{2} ; r_{1}^{\prime}, r_{2}^{\prime}\right)=\sum_{\mu} \frac{E_{\mu}\left(r_{1}, r_{2}\right) E_{\mu}^{*}\left(r_{1}^{\prime}, r_{2}^{\prime}\right)}{\omega-\omega_{\mu}}
$$

They are not the physical BFKL Green functions. The latter include an extra factor depending on $\mu$ :

$$
G\left(r_{1}, r_{2} ; r_{1}^{\prime}, r_{2}^{\prime}\right)=\frac{1}{4 \pi^{8}} \sum_{\mu} a_{n+1, \nu} a_{n-1, \nu} \frac{E_{\mu}\left(r_{1}, r_{2}\right) E_{\mu}^{*}\left(r_{1}^{\prime}, r_{2}^{\prime}\right)}{\omega-\omega_{\mu}}
$$

However in the limit $s \rightarrow \infty$ only the lowest conformal weights contribute $n=\nu=0$ for which $a_{n \pm 1, \nu}=2 \pi^{4}$ and (97) and (98) coincide. Then we can forget about tildas in (96).

We transform the Green functions to given total momenta of the pomerons presenting

$$
G_{3}\left(r_{1}, r_{2} ; r_{1}^{\prime}, r_{2}^{\prime}\right)=\int \frac{d^{2} l_{3}}{(2 \pi)^{2}} e^{i l_{3}\left(R_{3}-R_{3}^{\prime}\right)} G_{l_{3}}\left(r_{12}, r_{12}^{\prime}\right)
$$

where $R_{3}=(1 / 2)\left(r_{1}+r_{2}\right)$ and similarly for the two other Green functions. Introducing $R=r_{1}+r_{2}+r_{3}$ we transform the integration over the coordinates as follows

$$
\int d^{2} r_{1} d^{2} r_{2} d^{2} r_{3}=\int d^{2} R d^{2} r_{12} d^{2} r_{23} d^{2} r_{31} \delta^{2}\left(r_{12}+r_{23}+r_{31}\right)
$$

The coordinates themselves are $r_{1}=(1 / 3)\left(R-r_{21}-r_{31}\right)$ etc., wherefrom we find

$$
R_{1}=(1 / 6)\left(2 R-r_{12}-r_{13}\right), R_{2}=(1 / 6)\left(2 R-r_{21}-r_{23}\right), R_{3}=(1 / 6)\left(2 R-r_{31}-r_{32}\right)
$$

and

$$
i \sum_{j=1}^{3} l_{j} R_{j}=i(1 / 3) R \sum_{j=1}^{3} l_{j}-i(1 / 6)\left(r_{12} l_{12}+r_{23} l_{23}+r_{31} l_{31}\right)
$$


where we denoted $l_{12}=l_{1}-l_{2}$ etc. The integral over $R$ gives $9(2 \pi)^{2} \delta^{2}\left(l_{1}+l_{2}+l_{3}\right)$. Presenting the remaining $\delta$-function in (100) as an integral over an auxiliary momentum $q$ we find an expression (for fixed $l_{1}, l_{2}$ and $l_{3}$ )

$$
\begin{gathered}
\frac{g^{2} N_{c}}{8 \pi^{3}} \int \frac{d^{2} q}{(2 \pi)^{2}} \frac{d^{2} r_{12} d^{2} r_{23} d^{2} r_{31}}{r_{12}^{2} r_{23}^{2} r_{31}^{2}} \exp \left(i r_{12}\left(q-\frac{1}{6} l_{12}\right)+i r_{23}\left(q-\frac{1}{6} l_{23}\right)+i r_{31}\left(q-\frac{1}{6} l_{31}\right)\right. \\
G_{l_{3}}\left(r_{12}, r_{12}^{\prime}\right) G_{l_{1}}\left(r_{23}, r_{23}^{\prime}\right) G_{l_{2}}\left(r_{31}, r_{31}^{\prime}\right)
\end{gathered}
$$

At this point we recall the expression for the BFKL Green function with a fixed total momentum:

$$
G_{l}\left(r, r^{\prime}\right)=\frac{1}{(2 \pi)^{4}} \int \frac{\nu^{2} d \nu}{\left(\nu^{2}+1 / 4\right)^{2}} s^{\omega(\nu)} E_{\nu}^{(l)}(r) E_{\nu}^{(l)}\left(r^{\prime}\right)
$$

where

$$
E_{\nu}^{(l)}(r)=\int d^{2} R \exp (i l R)\left(\frac{r}{|R+r / 2||R-r / 2|}\right)^{1+2 i \nu}
$$

and where we retained only the dominant isotropic term. At $s \rightarrow \infty$ the vicinity of $\nu=0$ gives the dominant contribution. If $l \neq 0$ then the integral in (105) converges at large $R$ and we can take the functions $E$ out of the integral over $\nu$ at $\nu=0$. Taking then the asymptotics of the remaining integral, we find

$$
G_{l}\left(r, r^{\prime}\right) \simeq \frac{1}{2 \pi^{4}} s^{\Delta} \frac{\sqrt{\pi}}{(a \ln s)^{3 / 2}} E_{0}^{(l)}(r) E_{0}^{(l)}\left(r^{\prime}\right)
$$

where $\Delta=\omega_{n=0, \nu=0}$ is the BFKL intercept and $a=7 g^{2} N_{c} \zeta(3) /\left(2 \pi^{2}\right)$. As we see, the Green function asymptotically factorizes in $r$ and $r^{\prime}$. This means that we obtain a quantum field theory of pomerons with a propagator

$$
P(y, l)=\frac{2}{\pi^{2}} e^{y \Delta} \frac{\sqrt{\pi}}{(a y)^{3 / 2}}
$$

(not really depending on the momentum $l$ ) and an interaction vertex

$$
\begin{gathered}
V\left(l_{1}, l_{2}, l_{3}\right)=\frac{9 g^{2} N_{c}}{8 \pi^{3}} \int \frac{d^{2} q}{(2 \pi)^{8}} \frac{d^{2} r_{12} d^{2} r_{23} d^{2} r_{31}}{r_{12}^{2} r_{23}^{2} r_{31}^{2}} \\
\exp \left(i r_{12}\left(q-\frac{1}{6} l_{12}\right)+i r_{23}\left(q-\frac{1}{6} l_{23}\right)+i r_{31}\left(q-\frac{1}{6} l_{31}\right) E_{0}^{\left(l_{3}\right)}\left(r_{12}\right) E_{0}^{\left(l_{1}\right)}\left(r_{23}\right) E_{0}^{\left(l_{2}\right)}\left(r_{31}\right)\right.
\end{gathered}
$$

The vertex factorizes under the sign of the integration over $q$ :

$$
V\left(l_{1}, l_{2}, l_{3}\right)=\frac{9 g^{2} N_{c}}{8 \pi^{3}} \int \frac{d^{2} q}{(2 \pi)^{2}} J\left(l_{3}, q-\frac{1}{6} l_{12}\right) J\left(l_{1}, q-\frac{1}{6} l_{23}\right) J\left(l_{2}, q-\frac{1}{6} l_{31}\right)
$$

where

$$
J(l, q)=\int \frac{d^{2} r}{(2 \pi)^{2} r^{2}} e^{i q r} E_{0}^{(l)}(r)=\int \frac{d^{2} p}{2 \pi p} \frac{1}{|p-q+l / 2||p-q-l / 2|}
$$

Note that for $l=0$ this derivation is incorrect. Calculations show that in this case Eq. (109) for the vertex remains valid with

$$
J(0, q)=\frac{1}{9 q}
$$

However the Green function (104) at $l=0$ has an asymptotics

$$
G_{0}\left(r, r^{\prime}\right) \simeq \frac{1}{2 \pi^{2}} s^{\Delta} \sqrt{\frac{\pi}{a \ln s}} r r^{\prime} \exp \left(-\frac{\ln ^{2}\left(r / r^{\prime}\right)}{a \ln s}\right)
$$

so that the factorization is lost. 


\section{Conclusions}

Study of the 4-gluon system at $N_{c} \rightarrow \infty$ shows that in the leading order the system reduces to a single pomeron, as pointed out in [4]. The diffractive amplitude, subleading in $1 / N_{c}$, turns out to be a sum of the DPE and TPI contributions. The triple pomeron vertex which appears in the latter is different from the one introduced in [1] for $N_{c}=3$. However it is also conformal invariant. Moreover functions $G$ out of which both verteces are constructed are conformal invariant by themselves.

A novel feature of the diffractive amplitude in the hard pomeron model is the equivalence of the DPE and certain terms of TPI. This allows to eliminate the DPE contribution altogether and substitute it by additional TPI terms. The price of such a substitution is the appearance of some extra terms of a structure different from both the DPE and TPI, which are absent in the old Regge-Gribov theory.

The triple pomeron vertex obtained after this substitution coincides with a part $V(1234)$ of the vertex introduced in [1], leading in the high- colour limit. Coupling this vertex to two pomerons, most of the term vanish and the rest simple expression coincides with the triple pomeron vertex found by Peschanski in [5]. So there is a complete agreement between the results of $[1,3,5]$ in this respect. However the mentioned extra terms do not seem to appear in the dipole picture, which points to a certain difference between this approach and the $s$-channel unitarity one.

In the dipole approach the higher-order dipole densities are found to be represented by a set of pomeron fan diagrams with only a triple pomeron coupling. Four and more pomeron coupling do not appear, which is a clear prediction of the dipole picture. It would be interesting to verify this prediction in the framework of the $s$-channel unitarity approach by studying transitions from 1 to 3 pomerons.

\section{Acknowledgments.}

The authors express their deep gratitude to Prof G.Venturi for his constant interest in this work and helpful discussions. M.A.B. thanks the INFN and University of Bologna for their hospitality and financial help during his stay in Bologna.

\section{Appendix: Quark loop for non-zero momentum transfer}

Consider a $q \bar{q}$ loop for the scattering of a virtual photon $\gamma^{*}(q)+\ldots \rightarrow \gamma^{*}(q+l)+\ldots, q^{2}=-Q^{2}$. The momentum transfer $l$ is taken to be pure transversal. Then a straightforward calculation gives for the function $f(1,2)$ corresponding to the loop with gluon 1 attached to $q$ and gluon 2 attached to $\bar{q}$ the following expression

$$
f\left(k_{1}, k_{2}\right)=e_{f}^{2} \int_{0}^{1} d \alpha \int \frac{d^{2} k}{(2 \pi)^{3}} \frac{N}{D}
$$

where

$$
\begin{gathered}
D=\left(\epsilon^{2}+k^{2}\right)\left(\epsilon_{1}^{2}+\left(k+k_{1}-\alpha l\right)^{2}\right) \\
\epsilon^{2}=Q^{2} \alpha(1-\alpha)+m_{f}^{2}, \quad \epsilon_{1}^{2}=\left(Q^{2}+l^{2}\right) \alpha(1-\alpha)+m_{f}^{2}
\end{gathered}
$$

$e_{f}$ and $m_{f}$ are the quark electric charge and mass and the numerator for a transversal photon is

$$
N^{T}=m_{f}^{2}+\left(\alpha^{2}+(1-\alpha)^{2}\right) \mathbf{k}\left(\mathbf{k}+\mathbf{k}_{\mathbf{1}}\right)-\alpha^{2} \mathbf{k} \mathbf{l}
$$


and for a longitudinal photon is

$$
N^{L}=4 Q^{2} \alpha^{2}(1-\alpha)^{2}
$$

This expression can be conveniently represented as an integral over the colour dipole density $\rho$ created by the $q \bar{q}$ pair at a given distance in the transverse space:

$$
f\left(k_{1}, k_{2}\right)=\int d^{2} r \rho_{l}(r) e^{i k_{1} r}
$$

¿From (113) - (116) one finds for the transverse and longitudinal photons:

$$
\begin{gathered}
\rho_{l}^{T}(r)=\frac{e_{f}^{2}}{(2 \pi)^{3}} e^{-i \alpha l r} \int_{0}^{1} d \alpha\left[m_{f}^{2} K_{0}(\epsilon r) K_{0}\left(\epsilon_{1} r\right)+\right. \\
\left.\left(\alpha^{2}+(1-\alpha)^{2}\right) \epsilon \epsilon_{1} K_{1}(\epsilon r) K_{1}\left(\epsilon_{1} r\right)-\alpha(1-\alpha)(1-2 \alpha) \frac{i \epsilon \mathbf{l r}}{r} K_{0}\left(\epsilon_{1} r\right) K_{1}(\epsilon r)\right] \\
\rho_{l}^{L}(r)=\frac{4 e_{f}^{2} Q^{2}}{(2 \pi)^{3}} e^{-i \alpha l r} \int_{0}^{1} d \alpha \alpha^{2}(1-\alpha)^{2} K_{0}(\epsilon r) K_{0}\left(\epsilon_{1} r\right)
\end{gathered}
$$




\section{References}

1. J.Bartels and M.Wuesthoff, Z.Phys. C 66 (1995) 157.

2. J.Bartels, L.N.Lipatov and M.Wuesthoff, Nucl. Phys. B464 (1996) 298.

3. M.A.Braun, S.Petersburg preprint SPbU-97-9 (hep-ph/9706373).

4. M.A.Braun, Z.Phys. C71 (1996)601.

5. R.Peschanski, Saclay preprint, (hep-ph/9704342); Phys.Lett. 409 (1997) 491.

6. M.A.Braun, Santiago University preprint US-FT/4-94.

7. L.N.Lipatov, in "Perturbative QCD", ed. A.H.Mueller (World Sci., Singapore, 1989).

8. A.H.Mueller, Nucl.Phys. B415 (1994) 373; B437 (1995) 107;

A.H.Mueller and B.Patel, Nucl. Phys. B425 (1994) 471. 